\title{
Strong influence of packing density in terahertz metamaterials
}

\author{
Ranjan Singh, ${ }^{1,2, a)}$ Carsten Rockstuhl, ${ }^{3}$ and Weili Zhang ${ }^{1}$ \\ ${ }^{1}$ School of Electrical and Computer Engineering, Oklahoma State University, Stillwater, \\ Oklahoma 74078, USA \\ ${ }^{2}$ Center for Integrated Nanotechnologies, Materials Physics and Applications Division, Los Alamos \\ National Laboratory, Los Alamos, New Mexico 87545, USA \\ ${ }^{3}$ Institute of Condensed Matter Theory and Solid State Optics, Friedrich-Schiller-Universität Jena, \\ Jena 07743, Germany
}

(Received 3 August 2010; accepted 16 November 2010; published online 16 December 2010)

\begin{abstract}
We investigate the response of a terahertz metamaterial depending on the unit cell density. The fundamental inductive capacitive (LC) resonance has its highest quality $(Q)$ factor for a critical period $P c=\lambda / n$, with $\lambda$ being the LC resonance wavelength and $n$ being the refractive index of the substrate. This occurs due to simultaneous excitation of the lowest order lattice mode that strongly favors radiative coupling in the metamaterial plane. Increasing or decreasing the period from $P c$ reduces the $Q$ factor. Our results suggest that an optimal package density exists in metamaterials to induce the strongest dispersion. () 2010 American Institute of Physics. [doi:10.1063/1.3525169]
\end{abstract}

Metamaterials (MMs) constitute an exploding field of science and technology. It allows to mold the flow of electromagnetic waves almost arbitrarily using suitably tailored unit cells. Potentially the most common unit cell for MMs is the split ring resonator (SRR). ${ }^{1}$ The subwavelength closely packed SRR allows accessing several functionalities such as optical magnetism, negative refraction, frequency selection, modulation, sensing, and antenna capabilities. ${ }^{2-8}$ The impact on the light propagation depends strongly on the illumination and polarization direction with respect to the SRR. The unifying aspect in all such modi operandi is that the properties of the MM are predominantly determined by the resonant scattering response of light at individual SRRs. It is therefore essential to come up with schemes to maximize the quality $(Q)$ factor of the SRR resonances in order to optimize their performances for various applications. Moreover, a sharper resonance would lead to stronger dispersion in any effective parameter describing conceptually the MM. Since the $Q$ factor is limited by either radiative or nonradiative losses, their reduction is usually understood to be essential for achieving resonances with high $Q$ factors.

The $Q$ factor in symmetrical SRRs usually suffers from a strong coupling to free space radiation, resulting in high radiative losses. ${ }^{9}$ To overcome such problem, Fedotov et al. ${ }^{10}$ demonstrated that high $Q$ factors can be achieved by relying on dark modes. They are sustained by SRRs that are only weakly coupled to free space by a gentle break in symmetry. ${ }^{11-15}$ Nonradiative losses can be affected by controlling the distance between individual SRRs or cooling them to cryogenic temperatures ${ }^{16}$ to exploit superconductive properties ${ }^{17}$ of involved materials. A third aspect that affects the $Q$ factor is the radiative interaction of SRRs in the lattice. Such interaction was demonstrated in previous works by investigating the impact of disorder in the unit cell arrangement ${ }^{18-20}$ and the impact of laterally coupled $\mathrm{SRRs}^{21,22}$ on the resonance. Bitzer et al. ${ }^{23}$ showed that the inter-SRR coupling is mediated by lattice modes and it can have influence on the resonance sharpness. In another recent work Fedotov et al. $^{24}$ observed the collapse of a sharp Fano

${ }^{a)}$ Electronic mail: ranjan@lanl.gov. resonance with an increasing number of unit cell resonators but keeping the distance between the periodic metamolecules fixed. This observation was attributed to the suppression of radiative losses in periodic arrays. All these investigations point toward the importance of a proper tailoring of the radiative interaction among SRRs and their coupling to free space modes to enhance the overall $Q$ factor. The subtle impact of the SRR arrangement on the $Q$ factor can potentially be understood by considering the driving field of each SRR in the lattice. On one hand, the SRR is driven by the external illumination. On the other hand, it is driven by the scattered field from neighboring SRRs. If the distance between neighboring SRRs is too large, both fields tend to be out of phase, causing a decrease of the driving force since they interfere destructively. Changing the period changes the relative phase between the external illumination and the scattered light from other SRRs in the lattice leading to constructive or destructive interference and so to a modified driving force. The SRRs however should also not be placed too close since it favors nonradiative losses by capacitive and inductive coupling. Therefore, to obtain some final conclusions it is compulsory to investigate whether increasing or decreasing the number density of the SRR in the lattice has an effect on $Q$ factor of the fundamental resonance and to find an answer on what could be an optimal period to obtain the highest $Q$ factor.

In this letter, we demonstrate that the inter-SRR distance and its number density in a fixed area, so to say the period, can control the $Q$ factor and the strength of the inductive capacitive (LC) resonance of SRRs. We observe a strong suppression of radiative losses and thus a sharp enhancement of the $Q$ factor at a certain critical periodicity $P c$ that matches the SRR resonance wavelength normalized with the refractive index of the substrate. When the periodicity is further reduced, a gradual decline in $Q$ factor of the fundamental SRR resonance is observed. The minimal transmission in resonance shows an exponential decay as a function of the number density. Tailoring the periodicity of MMs will allow the design of frequency selective surfaces with an adjustable $Q$ factor for various applications and for optimized MMs across the entire electromagnetic spectrum. This finding will 

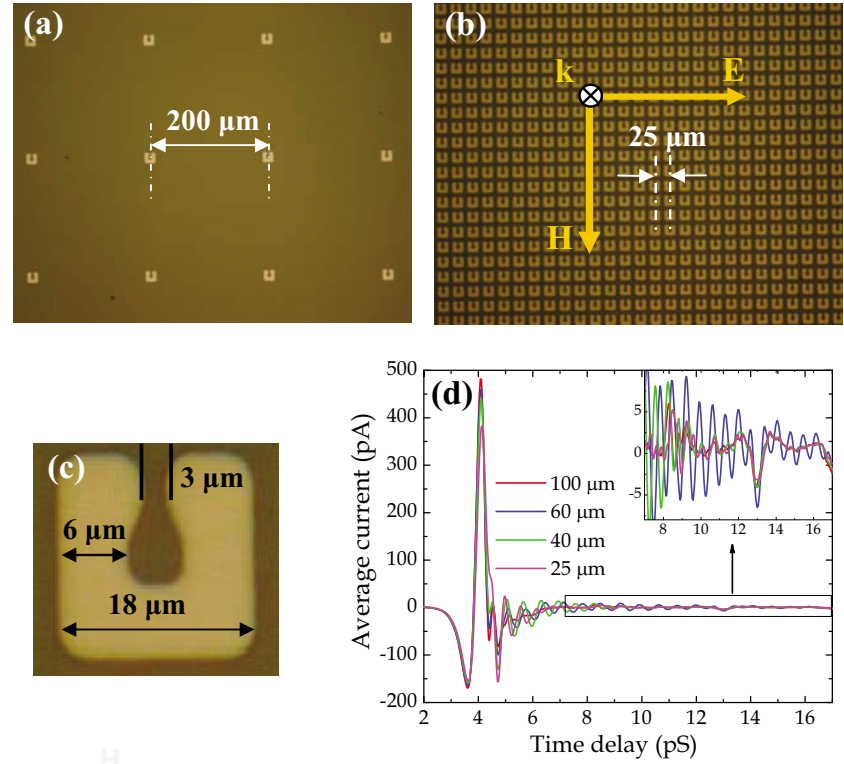

FIG. 1. (Color online) Microscopic image of the metamaterial array with (a) lowest packing density, $P=200 \mu \mathrm{m}$ and (b) highest packing density, $P$ $=25 \mu \mathrm{m}$, (c) unit cell with geometric dimensions, (d) measured transmitted pulses through different periodicity metamaterial.

eventually lead to the development of metamolecule density and substrate refractive index tuned terahertz devices and components.

In the experiments, we rely on a typical $8 f$ confocal photoconductive switch based terahertz time domain spectroscopy system consisting of a terahertz transmitter, beam collecting and steering optics, and a terahertz receiver. ${ }^{25,26}$ All the SRR metal film pattern was fabricated using photolithographic technique and then depositing $200 \mathrm{~nm}$ of aluminum metal on a $640 \mu \mathrm{m}$ thick n-type silicon substrate $(\varepsilon=11.68)$. The geometrical dimensions of the SRR are shown in Fig. 1(c). Retaining the identical structure of the SRR, nine sets of samples were prepared with periods of $P$ $=200,150,100,60,50,40,35,30$, and $25 \mu \mathrm{m}$. Figures 1 (a) and 1(b) show the MM sample with $P=200$ and $25 \mu \mathrm{m}$, respectively. The size of SRR was chosen such that its resonance wavelength is $\lambda=200 \mu \mathrm{m}$ for the silicon substrate. For samples with different periods, the resonance wavelength to lattice constant ratio $\lambda / P$ varies; ranging from a value of $\lambda / P=1$ for $P=200 \mu \mathrm{m}$ to $\lambda / P=8$ for $P=25 \mu \mathrm{m}$. Each $\mathrm{MM}$ sample is excited by an incident terahertz beam $3.5 \mathrm{~mm}$ in diameter at focus. Therefore, in the most diluted SRR sample with $P=200 \mu \mathrm{m}$, the number of SRRs being excited is around 960 and in the sample with the highest density where $P=25 \mu \mathrm{m}$, the number of SRRs being excited is 61500 . For a fixed area of terahertz beam excitation $\left[\pi(3.5 \mathrm{~mm})^{2} / 4\right]$, the density of SRRs is gradually increased from 960 to 61500 in order to probe the inter-SRR coupling and its impact on the overall resonant response of terahertz transmission. The MM sample size was kept fixed at $10 \mathrm{~mm} \times 10 \mathrm{~mm}$ and the incident terahertz field was polarized with the electric field parallel to the SRR gap at normal incidence [see Fig. 1(b)].

Figure 1(d) shows the measured time-domain transmission terahertz pulses through the samples with periodicity, $P=100,60,40$, and $25 \mu \mathrm{m}$ as they were illuminated at normal incidence exciting the fundamental LC resonance. We observe a significant reshaping of the terahertz pulse for different periodicities. A noteworthy observation is the high os-

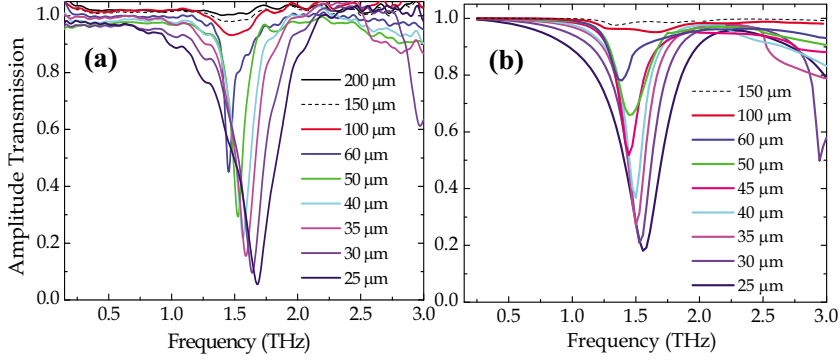

FIG. 2. (Color online) (a) Measured and (b) simulated transmission spectra of SRR arrays with varying periodicity.

cillation in the pulse for $P=60 \mu \mathrm{m}$. The transmission spectra in Fig. 2 are obtained by normalizing the measured transmission to the reference transmission of a blank n-type silicon wafer identical to the sample substrate for all the nine sets of MM. The evolution of the LC resonance can be observed at around $1.5 \mathrm{THz}$. The resonance was fairly weak for the 200,150 , and $100 \mu \mathrm{m}$ periodicities. A strong resonance feature is observed for a MM period of $60 \mu \mathrm{m}$ and then a gradual broadening of the resonance is seen for lower periodicities or higher packing densities. Simulations of the experiment based on the Fourier modal method reproduce the transmission measurement and are shown in Fig. 2. The $60 \mu \mathrm{m}$ periodicity corresponds to a $\lambda / P$ ratio of 3.33 that is close to the refractive index of the silicon substrate used in the experiment. This period will turn out to be the critical period. The measured transmission resonance minimum is plotted in Fig. 3(a). We observe that the transmission at the resonance exponentially decreases with the increasing density of metamolecules. The periodicity corresponding to the number of MM unit cells is plotted on the right hand $y$ axis of the same graph. Figure 3(b) indicates the strong dependence of the $Q$ factor on the chosen period. Although, especially for MMs with large periods, it is slightly difficult to evaluate the $Q$ factor very precisely, it can be seen that the $Q$ factor rises when the MM array reaches the optimum period and then declines with a further decreasing period.

The narrowest resonance occurs for a critical period, $P c=\lambda / n=60 \mu \mathrm{m}$. There, the lowest diffraction grating order in the substrate changes from being evanescent to being propagative. Principally, similar effects are anticipated to occur by evoking higher diffraction orders as well, although the dilution then needs to be increased and for practical reasons such higher diffraction orders are marginally relevant. The same holds for diffraction orders against the air cladding. In order to verify if the critical periodicity indeed allows observing the sharpest LC resonances, we conducted further simulations with different substrates while maintaining the

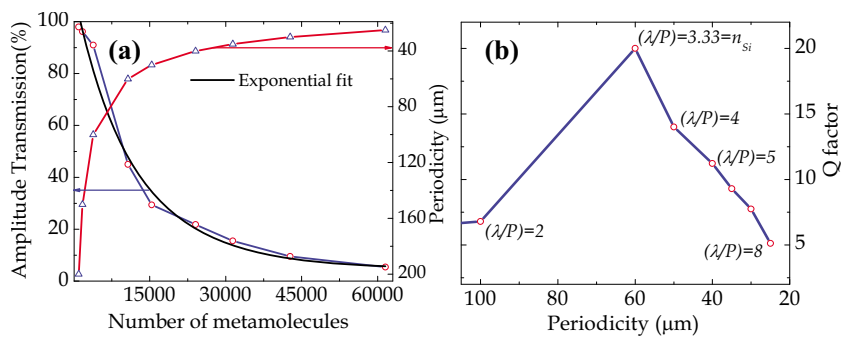

FIG. 3. (Color online) (a) Percentage transmission at resonance wavelength for different number of unit cells in a fixed area of terahertz exciting beam, (b) extracted $Q$ factor from experimental data for metamaterial with varying periodicity fabricated on silicon substrate with $n=3.33$. 


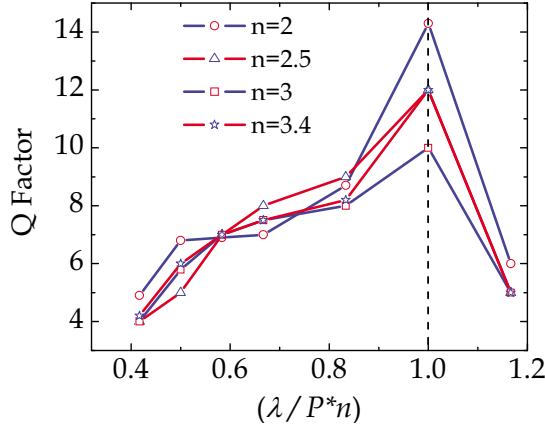

FIG. 4. (Color online) Simulated $Q$ factor of identical SRRs with different periodicities and different substrates indices.

dimensions of the SRR identical. The $Q$ factors extracted from the simulated transmission spectra for substrates with different indices are plotted in Fig. 4 depending on $\lambda / P^{*} n$. It can be clearly seen that the highest $Q$ factor is obtained whenever this normalized parameter is close to unity. It should be noted that with the change in the substrate index $n$, the resonance wavelength of the MM strongly changes and the absolute period at which the highest $Q$ factors are observed remains to be the same $(P c=60 \mu \mathrm{m})$. Thus, we define a MM to be an optimally packed MM when its periodicity is matched to the first order diffractive lattice mode at $P c=\lambda / n$. The samples with higher periodicity than $P c$ or with $P c>\lambda / n$ can be termed as loosely packed $\mathrm{MM}$ and the ones with lower periodicity than $P c$ or with $P c<\lambda / n$ can be defined as tightly packed MM.

The narrowing of the resonance in an optimally packed MM can potentially be best understood by the suppression of radiation damping due to the confinement or trapping of electromagnetic fields in the MM array. This mechanism is based on diffractive coupling during which the diffracted fields are trapped in the MM lattice and each SRR radiatively couples to the neighboring SRR. ${ }^{23,27}$ The lower $Q$ factor for larger periodicity is associated to an enhanced damping and for smaller periodicity, it is mainly due the mismatch with lattice mode during which the radiated fields of the coherently coupled SRRs destructively interfere in the plane of the MM leading to higher radiative losses and the increase of nonradiative losses due to capacitive and inductive coupling. The blueshifting of the resonance is potentially due to the near field transverse magnetic dipole coupling between individual SRRs. The broadening is caused by an increase in radiative damping that effectively reduces the cross section per SRR.

In summary, we have experimentally and numerically demonstrated that the MM arrays have the best resonance response for an optimum lattice periodicity that allows strong radiative coupling among the SRRs mediated by the lattice modes. This strongly depends on the resonance wavelength of SRRs and the substrate refractive index. Loosely or tightly packed MMs favor radiative damping, leading to broadening of the fundamental LC resonance and thus a de- cline in the $Q$ factor. The dependence of the $Q$ factor on the distance between the SRRs provides a method for designing subwavelength terahertz MM cavities with an adjustable $Q$ factor.

The authors would like to thank Christoph Menzel for contiuous support in simulations, Markus Walther for constructive comments, D. R. Chowdhury, M. T. Reiten, and J. Zhou for fruitful discussions. This work was partially supported by the U.S. National Science Foundation Grant No. ECCS-0725764, and the German Federal Ministry of Education and Research (Metamat) and the DAAD within the PPP program.

${ }^{1}$ J. B. Pendry, A. Holden, D. Robbins, and W. Stewart, IEEE Trans. Microwave Theory Tech. 47, 2075 (1999).

${ }^{2}$ R. A. Shelby, D. R. Smith, and S. Schultz, Science 292, 77 (2001).

${ }^{3}$ T. J. Yen, W. J. Padilla, N. Fang, D. C. Vier, D. R. Smith, J. B. Pendry, D. N. Basov, and X. Zhang, Science 303, 1494 (2004).

${ }^{4}$ C. M. Soukoulis, S. Linden, and M. Wegener, Science 315, 47 (2007).

${ }^{5}$ H. T. Chen, W. J. Padilla, J. M. O. Zide, A. C. Gossard, A. J. Taylor, and R. D. Averitt, Nature (London) 444, 597 (2006).

${ }^{6}$ J. F. O'Hara, R. Singh, I. Brener, E. Smirnova, J. Han, A. J. Taylor, and W. Zhang, Opt. Express 16, 1786 (2008).

${ }^{7}$ R. Singh, C. Rockstuhl, C. Menzel, T. P. Meyrath, M. He, H. Giessen, F. Lederer, and W. Zhang, Opt. Express 17, 9971 (2009).

${ }^{8}$ W. Withayachumnankul and D. Abbott, IEEE Photonics J. 1, 99 (2009).

${ }^{9}$ R. Singh, A. K. Azad, J. F. O'Hara, A. J. Taylor, and W. Zhang, Opt. Lett. 33, 1506 (2008).

${ }^{10}$ V. A. Fedotov, M. Rose, S. L. Prosvirnin, N. Papasimakis, and N. I. Zheludev, Phys. Rev. Lett. 99, 147401 (2007).

${ }^{11}$ R. Singh, C. Rockstuhl, F. Lederer, and W. Zhang, Phys. Rev. B 79, 085111 (2009).

${ }^{12}$ N. Liu, L. Langguth, T. Weiss, J. Kastel, M. Fleischhauer, T. Pfau, and H. Giessen, Nature Mater. 8, 758 (2009).

${ }^{13}$ R. Singh, E. Plum, W. Zhang, and N. I. Zheludev, Opt. Express 18, 13425 (2010).

${ }^{14}$ R. Singh, E. Plum, C. Menzel, C. Rockstuhl, A. K. Azad, R. A. Cheville, F. Lederer, W. Zhang, and N. I. Zheludev, Phys. Rev. B 80, 153104 (2009)

${ }^{15}$ R. Singh, I. A. I. Al-Naib, M. Koch, and W. Zhang, Opt. Express 18, 13044 (2010).

${ }^{16}$ R. Singh, Z. Tian, J. Han, C. Rockstuhl, J. Gu, and W. Zhang, Appl. Phys. Lett. 96, 071114 (2010).

${ }^{17}$ J. Gu, R. Singh, Z. Tian, W. Cao, Q. Xing, M. He, J. W. Zhang, J. Han, H.-T. Chen, and W. Zhang, Appl. Phys. Lett. 97, 071102 (2010).

${ }^{18}$ N. Papasimakis, V. A. Fedotov, Y. H. Fu, D. P. Tsai, and N. I. Zheludev, Phys. Rev. B 80, 041102(R) (2009).

${ }^{19}$ C. Helgert, C. Rockstuhl, C. Etrich, C. Menzel, E. B. Clay, A. Tunnermann, F. Lederer, and T. Pertsch, Phys. Rev. B 79, 233107 (2009).

${ }^{20}$ R. Singh, X. Lu, J. Gu, Z. Tian, and W. Zhang, J. Opt. 12, 015101 (2010)

${ }^{21}$ R. Singh, C. Rockstuhl, F. Lederer, and W. Zhang, Appl. Phys. Lett. 94, 021116 (2009).

${ }^{22}$ N. Feth, M. Konig, M. Husnik, K. Stannigel, J. Niegemann, K. Busch, M. Wegener, and S. Linden, Opt. Express 18, 6545 (2010).

${ }^{23}$ A. Bitzer, J. Wallauer, H. Helm, H. Merbold, T. Feurer, and M. Walther, Opt. Express 17, 22108 (2009).

${ }^{24}$ V. A. Fedotov, N. Papasimakis, E. Plum, A. Bitzer, M. Walther, P. Kuo, D. P. Tsai, and N. I. Zheludev, Phys. Rev. Lett. 104, 223901 (2010).

${ }^{25}$ A. K. Azad, J. Dai, and W. Zhang, Opt. Lett. 31, 634 (2006).

${ }^{26}$ D. Grischkowsky, S. Keiding, M. van Exter, and Ch. Fattinger, J. Opt. Soc. Am. B 7, 2006 (1990).

${ }^{27}$ V. G. Kravets, F. Schedin, and A. N. Grigorenko, Phys. Rev. Lett. 101, 087403 (2008). 